\title{
Medical students now choosing wisely
}

$\mathrm{M}$ edical students across Canada are challenging what they describe as a culture of waste in medical education. Thirty-four student leaders from Canada's 17 medical schools met in Toronto on Nov. 14 to brainstorm ways to raise awareness about the harms of "more-is-better" medicine.

The summit was hosted by Choosing Wisely Canada, a campaign that issues lists of often unnecessary tests and treatments that doctors should think twice about ordering. Now, the campaign is supporting students to launch grassroots initiatives based on a new list of potentially wasteful behaviours that trainees should question, including "ordering tests or treatments for the sole purpose of gaining clinical experience."

According to Dr. Wendy Levinson, chair of Choosing Wisely Canada, medical education often rewards students who demonstrate their knowledge and efficiency by "ordering everything, often pre-emptively," regardless of potential harms to patients. "There's a culture of leaving no stone unturned," Levinson says. "Students learn that a good trainee thinks of all the possible reasons for a patient's chest pain, from the very likely to the completely improbable, and then orders all the associated tests to show that they've thought of them."

There's growing evidence that doctors carry over these wasteful habits from training into practice, Levinson adds. "We've taken the approach that trying to change the faculty is maybe too difficult ... so let's engage with the students and let them educate the faculty."

Choosing Wisely Canada selected students to attend the Toronto summit based on their leadership background and proposals for local campaigns. Proposed initiatives ranged from mentorship programs to journal clubs to hosting conferences. "One student who worked in Silicon Valley before going to medical school wanted to create an online game," noted Karen Born, knowledge translation lead for Choosing Wisely Canada. "Really the sky is

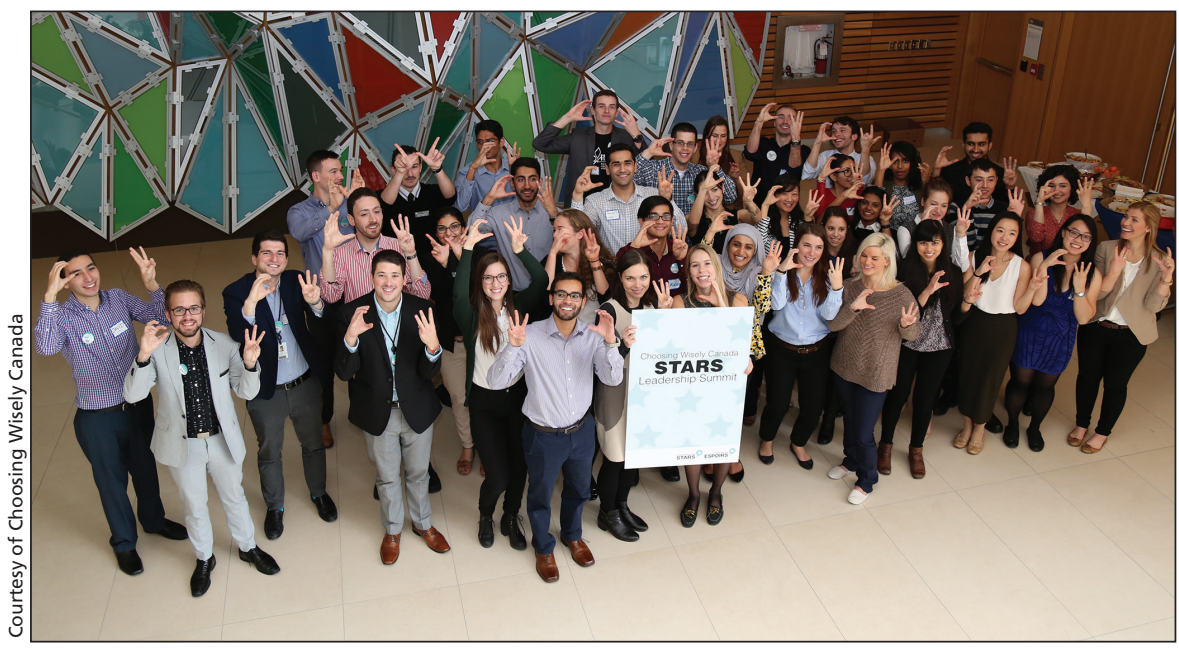

Medical students are challenging supervisors about unnecessary tests and treatments.

the limit in terms of what these students do."

The model is already working at the University of Toronto, where students have motivated professors to add slides on relevant Choosing Wisely recommendations to their lectures. But even Choosing Wisely converts say it's difficult to resist the pressure to order first, ask questions later. "You want to please the staff and do what they think is best," explains William Silverstein, a third-year medical student at the University of Toronto.

Contrary to his fears, however, Silverstein has found that asking questions about the usefulness of certain tests "almost gives you brownie points ... because it reflects that I've been doing the reading and I'm clearly dedicated to the patient."

Anand Lakhani, a second-year medical student at the University of Toronto, codeveloped the new Choosing Wisely recommendations for trainees with Silverstein. "Everyone we've spoken to is dealing with the pressures of the hierarchy of medical education," says Lakhani. "Hopefully this list can give them support to start asking questions."

According to the list, medical students and trainees should not:

- order the most invasive test or treatment before considering less invasive options;
- suggest a test, treatment or procedure that will not change a patient's clinical course;

- miss the opportunity to initiate conversations with patients about whether a test, treatment or procedure is necessary;

- hesitate to ask for clarification on tests, treatments or procedures that they believe are unnecessary;

- suggest ordering tests or performing procedures for the sole purpose of gaining clinical experience; or

- order tests or treatments pre-emptively for the sole purpose of anticipating what your supervisor would want.

Parallel to student efforts, however, "medical school curricula will need to evolve to more explicitly teach these concepts," says Dr. Brian Wong, medical education lead for Choosing Wisely Canada.

The return to the days where "we didn't always need to order tests to understand how to look after patients" resonates with educators from eras when there wasn't easy access to tests, he adds. Meanwhile, "my younger colleagues get frustrated with the waste they see in the health care system." - Lauren Vogel, CMAJ

CMAJ 2016. DOI:10.1503/cmaj.109-5198 